\title{
HS-SPME/GC-MS analysis of volatile and semi-volatile organic compounds emitted from municipal sewage sludge
}

\author{
Urszula Kotowska $\cdot$ Maciej Żalikowski • \\ Valery A. Isidorov
}

Received: 21 April 2010 / Accepted: 25 May 2011 / Published online: 18 June 2011

(C) The Author(s) 2011. This article is published with open access at Springerlink.com

\begin{abstract}
The aim of the research involved identification and semi-quantitative determination of unknown volatile and semi-volatile organic compounds emitted to air by sewage sludge formed in the process of municipal wastewater treatment in a sewage treatment plant. Samples taken directly after completion of the technological process as well as the sludge stored on the premise of the sewage treatment plant were analyzed. A simple method using off-line headspace solid-phase microextraction combined with gas chromatography-mass spectrometry has been proposed for extraction and detection of organic pollutants. For reliable identification of compounds, combination of two independent parameters: mass spectra and linear temperature programmed retention indices were employed. Over 170 compounds of different structure were identified including aliphatic and aromatic hydrocarbons, alcohols, esters, carbonyls, as well as sulfur, nitrogen, and chlorine containing compounds. The prevailing substances included: ethyl ether, $n$-hexane, $p$-xylene, $o$-xylene, mesitylene, $m$-ethylbenzene, limonene, $n$-decane, $n$-undecane, and $n$-dodecane. A few compounds such as methanetiol, dimethyl polisulfide, octaatomic
\end{abstract}

\footnotetext{
U. Kotowska $(\bowtie) \cdot$ M. Żalikowski · V. A. Isidorov Institute of Chemistry, University of Bialystok, ul. Hurtowa 1, 15-399 Bialystok, Poland e-mail: ukrajew@uwb.edu.pl
}

sulfur, phthalic anhydride, and indoles were identified in the sludge for the first time.

Keywords Sewage sludge • Organic compounds • HS-SPME • GC-MS

\section{Introduction}

Approached quantitatively, sludge formed in the process of wastewater treatment constitutes a few percent of waste products produced by human economic activity. However, due to the environmental threat, it poses makes sewage sludge management one of the most difficult problems in the majority of European countries. According to the policy of the European Council, the most desired solution to the problem of sewage sludge management is sludge recycling, i.e., its return to the environment with the use of natural methods, including agricultural activities. The indispensable condition of the implementation sludge recycling is to assure appropriate sludge quality including both biological (i.e., elimination of widely occurring in sludge pathogenic organisms, such as pathogenic bacteria, fungi, and eggs of human and animal parasites) as well as chemical purity.

Volatile organic compounds (VOC) and semivolatile organic compounds (SVOC) contained in sewage and sewage sludge can exert a harmful influence on the natural environment and human 
health. Certain groups of these compounds reach sewage treatment plants in the wastewater from households and industrial plants. Some of them undergo biodegradation during the process of sewage treatment; others remain in the sewage sludge in an unchanged form. A large number of VOCs and SVOCs are formed during decomposition of various organic compounds as well as during metabolism of microorganisms that form active sludge in biological sewage treatment plants. Due to their volatile character, some of these compounds escape to the atmosphere at different stages of sewage treatment processes (Simonich et al. 2000; Escalasa et al. 2003; Wu et al. 2006).

In the literature on the subject, only a small number of research papers has been devoted to the determination of volatile and semi-volatile organic compounds in the sewage sludge. Main VOCs identified in the sewage sludge are: $n$ alcanes, branched, cyclic, and unsaturated aliphatic hydrocarbons, aromatic hydrocarbons, and halogenated aliphatic hydrocarbons (Eitzer 1995; Wilson et al. 1996a, b; Pagans et al. 2006; Harrison et al. 2006; Koe and Shen 1997; Webber et al. 1996; Van Durme et al. 1992). Dimethyl sulfide, dimethyl disulfide, limonene, and $\alpha$-pinene were identified as the most significant odorous VOCs in the wastewater sludge. Other compounds included: carbon disulfide (Eitzer 1995; Harrison et al. 2006), carbonyl compounds (Eitzer 1995; Wilson et al. 1996a), and some terpenes: camphene, 3-carene, terpineol (Eitzer 1995). The most frequently detected SVOCs in the sludges were: polycyclic aromatic hydrocarbons, phthalic acid esters, chlorobenzenes, nitroaromatic compounds, and phenols (Harrison et al. 2006; Cai et al. 2007).

Extraction and concentration of volatile and semi-volatile organic compounds from the samples of sludge were made with the use of sorption tubes filled up with Tenax, Carbotrap, or Carbosieve granulates (Eitzer 1995; Koe and Shen 1997). The sampling using sorbent tubes or traps requires complete retention during sampling as well as complete recovery during analysis. Quantitative monitoring of vapor-phase organics using sorbents could be made by active sampling onto tubes packed with one or more sorbents, passive sampling onto sorbents or cartridges or whole air sampling methods (Matysik et al. 2009; Woolfenden 2010a, b; ISO EN 2000; ISO EN 2003; ASTM 1998; NSAI 2011; Kim et al. 2005). For the recovery of analytes from sorbents, two techniques: solvent extraction or thermal desorption are used (ISO EN 2000; ISO EN 2003; US EPA 1996). For the separation of VOCs and SVOCs from sludge samples, the "purge and trap" technique (Webber et al. 1996) and extraction with water-pentane mixture (Wilson et al. 1996b) are applied. The gaseous phase can also be taken directly with the help of a gas-proof syringe ( $\mathrm{Pa}-$ gans et al. 2006). For the final determination the technique of gas chromatography coupled with the detection with the mass spectrometry technique (GC-MS) or with a flame ionisation detector (GC-FID) is performed.

In this work, to extract both VOCs and SVOCs from wastewater sludge, the SPME technique has been implemented. This technique, devised in 1987-1989 (Pawliszyn and Liu 1987; Arthur and Pawliszyn 1990) is a simple and effective extraction technique that eliminates the need of using solvents or complicated apparatus. To conduct SPME isolation, a relatively small sample is required and its preparation is quick. In direct immersion SPME (DI-SPME), the fiber is directly placed in the sample. In headspace SPME mode, the fiber is exposed to the vapor phase above the liquid or solid matrix. Headspace solid-phase microextraction (HS-SPME) is frequently used for isolation of volatile and semi-volatile compounds (Pawliszyn 1997). Desorption of compounds retained on the fiber may be conducted directly in a GC injector. Although this desorption manner may be loaded with some risk of introducing air to the system and a resultant loss of most volatile compounds, it is the most commonly used method due to its simplicity and no need to use additional equipment.

SPME is a microextraction technique, which means that the amount of extraction medium is very small compared to the sample volume. As a result, exhaustive transfer of analytes to the extracting phase, which is characterized by the retention of vapor-organics on sorbents, does not occur here. HS-SPME is a complex multi-phase partitioning system, where equilibrium is reached in two systems: between the sample matrix and 
the headspace and between the headspace and the extracting phase. Temperature exerts a great impact on those processes. Also other experimental conditions greatly affect the course of the isolation process, e.g., extraction time, sample mixing, sample volume, headspace volume, $\mathrm{pH}$, salting out effect, etc. Strict adherence to the procedures established for SPME technique is more important than equilibrium conditions during the extraction.

The HS-SPME technique has been widely used for the determination of VOCs and SVOCs present in various matrices. SPME, in combination with GC and HPLC, was reported to have been used for the analysis of VOCs and SVOCs in indoor air (Hippelein 2006), lower troposphere (Mangani et al. 2003), gaseous industrial effluents (Domeño et al. 2004), and human breath (Spinhirne et al. 2004). Volatile and semi-volatile organic compounds were extracted with the SPME technique also from water samples (Bravo-Linares et al. 2007) and biological samples: plant and animal tissues and organisms (Isidorov et al. 2005a, b; Villaverde et al. 2007), bacteria (Gu et al. 2007), and blood (Blount et al. 2006).

There are only a few papers dealing with the application of SPME to the analysis of organic pollutants in sludge samples. DI-SPME was used for the determinsation of nitrobenzenes, nitrotoluene, and triazines in sludge samples (Basheer and Lee 2004; Jönsson et al. 2007; Zeng et al. 2009). Synthetic polycyclic musks were extracted from sewage sludges by HS-SPME and microwave-assisted SPME (Llompart et al. 2003; Wu and Ding 2010) during aliphatic primary amines isolation by pressurized hot water extraction followed by HS-SPME (Llop et al. 2010).

\section{Experimental section}

Sludge samples Samples of sludge were obtained from the Wastewater Treatment Plant located in Białystok. The plant receives municipal wastewater from the area of Białystok Cityapproximately 15 million cubic meters of wastewater are disposed to the plant in a year. The treatment process consists of mechanical and biological stages and lasts from 24 to $30 \mathrm{~h}$. The sludge, being a by-product of the technological process of the sewage treatment, is dehydrated and stored on a heap. The sewage sludge is composed of excessive active deposit mixed with suspension coming from the wastewater reaching the sewage treatment plant and stored in preliminary settling tanks. Sewage sludge samples were collected eight times between October 2006 and May 2007. The samples were collected from the dehydrating press to a glass container and analyzed immediately after collection.

VOC and SVOC sampling Isolation of VOCs was carried out with the help of SPME fibers with coating of Carboxene suspended in polidimethylsiloxane (CAR/PDMS, $75 \mu \mathrm{m})$ and fibers with dual coating of divinylbenzene and carboxene $(50 / 30 \mu \mathrm{m})$ suspended in polidimethylsiloxane (DVB/CAR/PDMS) made by Supelco, USA. Extraction of VOCs and SVOCs was carried out in both laboratory and field conditions. In the first variant a hermetic glass container of $900 \mathrm{ml}$ in volume fitted with inlet ports was filled with $150 \mathrm{~g}$ sludge with the addition of $700 \mathrm{ml}$ of deionized water. Next, the SPME device fiber, previously conditioned according to manufacturer's recommendations, was inserted into the headspace above the sample. The extraction was carried out for $2.5 \mathrm{~h}$ at $20^{\circ} \mathrm{C}$ with mixing speed of $900 \mathrm{rpm}$. In the field conditions, the sampling of volatiles was carried out using poly(methyl methacrylate) chamber placed on the dehydrated sludge. The chamber was fitted with two inlet ports for SPME fiber and a fan ensuring continuous mixing of gases inside the chamber. The extraction was carried out on the heap situated in the area of the sewage treatment plant in Białystok, where the sludge is transferred after dehydration. The sludge transferred from the dehydrating press is continuously mixed with the sludge stored on the heap, which gives its entire volume the same properties. The sampling chamber was positioned on the surface of such averaged sludge. The sampling was conducted three times between November 2006 and April 2007; during experiments the air temperature ranged from $0^{\circ} \mathrm{C}$ to $10^{\circ} \mathrm{C}$, the temperature of the sludge was ca. $20^{\circ} \mathrm{C}$. The extraction was carried out for $2.5 \mathrm{~h}$. After this time the SPME device fibers were protected with silicone septa and transferred to the laboratory, where the 
analytes were instantly desorbed thermally in an injector of gas chromatograph and analysed using the GC-MS technique.

Instrumentation Gas chromatographic analyses were carried out using a HP 6890 gas chromatograph with electronic pressure control and with a mass selective detector HP 5973 (electron impact source and quadrupole analyzer) from the Agilent Technologies, USA. This device was equipped with split/splitless injector and HP-5MS column (5\% phenylmethylsiloxane) size $30 \mathrm{~m} \times 0.25 \mathrm{~mm}$, i.e., coated with $0.25 \mu \mathrm{m}$ film thickness. The scan frequency was 2.64 times per second and the mass range scanned was 28-600 amu. The carrier gas was helium $(99.999 \%)$ at a constant flow rate of $1.0 \mathrm{ml} / \mathrm{min}$. A splitless injection technique was used and injection port temperature of $250^{\circ} \mathrm{C}$. The oven program was as follows: $35^{\circ} \mathrm{C}$ for $5 \mathrm{~min}$, $3^{\circ} \mathrm{C} / \mathrm{min}$ to $300^{\circ} \mathrm{C}, 300^{\circ} \mathrm{C}$ for $15 \mathrm{~min}$. The electron impact source temperature was $230^{\circ} \mathrm{C}$ with electron energy of $70 \mathrm{eV}$. The quadrupole temperature was $150^{\circ} \mathrm{C}$, and $\mathrm{GC}$ interface temperature was $280^{\circ} \mathrm{C}$. The instrument was tuned on perfluorotributylamine.

\section{Results and discussion}

Table 1 contains a list of organic compounds extracted from the sewage sludge, as well as some analytical parameters used for identification, Chemical Abstracts Service Registry Number for each compound and information on the variant of determination, where a given compound has been identified. Exemplary chromatograms are presented in Fig. 1.

Before commencing our research, the amount of information concerning the composition of organic compounds contained in the sewage sludge was rather poor, due to the limited number of publications concerning this problem. Additionally, the composition of the sewage sludge depends on some local factors, such as the number and type of industrial plants connected to the sewage treatment plant and the management of wastewater treatment processes. Taking the above into account, the research was mainly focused on prospective analyses. To ensure correct identification results, two independent parameters, i.e., mass spectra and linear temperature programmed chromatographic retention indices were applied.

Mass spectra recorded during the analyses were compared to spectra contained in the NIST MS base. Using the equation given by Van den Dool and Kratz (1963), linear temperature programmed retention indices (LTPRI) values were calculated on the basis of the chromatograms of samples as well as the chromatograms recorded for the mixture of $C_{8}-C_{40} n$-alkanes. Next, the LTPRI values were compared to indexes available in catalogs (de Zeeuw et al. 1992; Sadtler Research Laboratory 1986; Adams 1995; NIST Chemistry WebBook, http://webbook.nist.gov/chemistry/) and in our previous publication (Isidorov et al. 2005a, b). The names of all compounds fully identified are shown in Table 1 with no additional marks. It means that mass spectral data matched library spectra, and GC retention index data matched library index. The compounds tentatively identified, marked with (a), refer to identities whose spectral data matched those found in the library; however, their retention index values could not be found in the literature, or their determination was not possible.

All identified compounds were categorized into the following groups: aliphatic hydrocarbons, aromatic hydrocarbons, terpenes, sulfur compounds, ethers, alcohols, chloride compounds, esters, carbonyls, nitrogen-containing compounds, and nonidentified compounds. In the course of laboratory research, 164 organic compounds of various structures were registered. The substances in largest amounts found in the laboratory research included ethyl ether, $n$-hexane, $o, p$-xylene, mesitylene, $m$-ethylbenzene, limonene, $n$-decane, $n$-undecane, and $n$-dodecane. The most numerous group of compounds were aliphatic and aromatic hydrocarbons. In the group of aliphatic hydrocarbons, compounds of saturated character and linear structure ( $n$-alkanes $\left.C_{8}-C_{33}\right)$ and branched compounds (methylnonane, methyldecane, methylundecane, etc.) were predominating. The relative percentage (calculated as a fraction of the total ion current, TIC quotient between the sum of peak areas of compounds belonging to a particular group and the sum of peak areas of all 
Table 1 Identification results of organic compounds emitted from municipal sludges and values of parameters used in identification process

\begin{tabular}{|c|c|c|c|c|c|c|}
\hline Compound & CAS\# & $L T P R I^{\operatorname{Exp}}$ & LTPRI $I^{\text {Lit }}$ & $m / z$ & $\mathrm{M}^{\bullet+}$ & Occurrence \\
\hline Methyl alcohol $^{\mathrm{a}}$ & $67-56-1$ & - & 381 & $31,29(73), 30$ & 32 & $\mathrm{w}, \mathrm{y}, \mathrm{z}$ \\
\hline Methanethiol $^{\mathrm{a}}$ & $74-93-1$ & - & - & $47,48(80), 45(51), 46$ & 48 & $\mathrm{w}, \mathrm{x}, \mathrm{y}$ \\
\hline Ethanol $^{\mathrm{a}}$ & $64-17-5$ & - & 448 & $31,45(75), 27(46), 46,55$ & 46 & $\mathrm{w}, \mathrm{x}, \mathrm{z}$ \\
\hline Acetone $^{\mathrm{b}}$ & $67-64-1$ & 500 & 500 & $43,58(37), 41(11), 42,39$ & 58 & $\mathrm{w}, \mathrm{x}, \mathrm{y}, \mathrm{z}$ \\
\hline Trimethylamine $^{\mathrm{a}}$ & $75-50-3$ & - & 503 & $58,59(50), 42(34), 43$ & 59 & $\mathrm{z}$ \\
\hline Ethyl ether & $60-29-7$ & 510 & 509 & $31,59(88), 74(70), 45,29$ & 74 & $\mathrm{w}, \mathrm{x}, \mathrm{y}, \mathrm{z}$ \\
\hline Dimethyl sulfide & $75-18-3$ & 521 & 514 & $62,47(75), 45(45), 46,61$ & 62 & $\mathrm{w}, \mathrm{y}$ \\
\hline Methylene chloride ${ }^{b, c}$ & $75-09-2$ & 531 & 528 & $86,84(73), 49(65), 51,29$ & 84 & $\mathrm{w}$ \\
\hline Carbon disulfide $^{\mathrm{b}}$ & $75-15-0$ & 536 & 540 & $76,78(9), 44(9), 38$ & 76 & $\mathrm{w}, \mathrm{x}, \mathrm{y}, \mathrm{z}$ \\
\hline$n$-Hexane & $110-54-3$ & 600 & 600 & $57,41(66), 43(56), 56,42$ & 86 & $\mathrm{w}, \mathrm{x}, \mathrm{y}, \mathrm{z}$ \\
\hline 2-Butanone ${ }^{b}$ & $78-93-3$ & 602 & 602 & $43,72(32), 29(15), 57,27$ & 72 & $\mathrm{y}$ \\
\hline $\begin{array}{l}\text { Ethene, 1,2-dichloro-, } \\
\text { (Z)-a,b,c }\end{array}$ & $156-59-2$ & 608 & - & $96,61(91), 98(64), 63,60$ & 96 & w \\
\hline Ethyl acetate & $141-78-6$ & 612 & 607 & $43,61(21), 70(17), 45,29$ & 88 & $\mathrm{w}, \mathrm{x}, \mathrm{y}, \mathrm{z}$ \\
\hline Trichloromethane $e^{b, c}$ & $67-66-3$ & 615 & 616 & $83,85(62), 47(16), 87,48$ & 118 & W \\
\hline Benzene $^{\mathrm{b}, \mathrm{c}}$ & $71-43-2$ & 653 & 657 & $78,77(23), 51(12), 52,50$ & 78 & $\mathrm{w}, \mathrm{x}, \mathrm{y}$ \\
\hline 3-Pentanone & $96-22-0$ & 689 & 688 & $43,41(25), 86(20)$ & 86 & $\mathrm{w}$ \\
\hline$n$-Heptane & $142-82-5$ & 700 & 700 & $43,28(60), 41(59), 57,71$ & 100 & $\mathrm{w}, \mathrm{x}$ \\
\hline Trichloroethylene $\mathrm{e}^{\mathrm{b}, \mathrm{c}}$ & $79-01-6$ & 701 & 702 & $132,95(85), 130$ & 130 & w \\
\hline Bromodichloromethane $e^{b, c}$ & $75-27-4$ & 706 & 709 & $83,85(66)$ & - & w \\
\hline Furan, 2,5-dimethyl- & $625-86-5$ & 706 & 712 & $96,95(80), 43(39), 53,81$ & 96 & $\mathrm{w}$ \\
\hline Disulfide, dimethyl & $624-92-0$ & 735 & 733 & $94,79(40), 45,40$ & 94 & $\mathrm{w}, \mathrm{y}, \mathrm{z}$ \\
\hline Pyridine & $110-86-1$ & 736 & 753 & $79,52(47), 51(24), 50,78$ & 79 & $\mathrm{w}, \mathrm{x}, \mathrm{z}$ \\
\hline Toluene $\mathrm{e}^{\mathrm{b}, \mathrm{c}}$ & $108-88-3$ & 757 & 760 & $91,92(64), 65(10), 39,93$ & 92 & $\mathrm{w}, \mathrm{x}, \mathrm{y}, \mathrm{z}$ \\
\hline Thiophene, 3-methyl- & $616-44-4$ & 784 & 786 & $97,98(54), 45,51$ & 98 & $\mathrm{~W}$ \\
\hline 3-Pentanone, 2,4-dimethyl- & $565-80-0$ & 794 & 794 & $43,71(46), 41(28)$ & 114 & $\mathrm{w}$ \\
\hline$n$-Octane & $111-65-9$ & 800 & 800 & $43,85(84), 41(82), 57,71$ & 114 & $\mathrm{w}, \mathrm{x}$ \\
\hline Tetrachloroethylene $e^{b, c}$ & $127-18-4$ & 806 & 814 & $166,164(82), 129(69), 131,168$ & 164 & w \\
\hline Ethylbenzene $\mathrm{e}^{\mathrm{b}, \mathrm{c}}$ & $100-41-4$ & 857 & 850 & $91,106(38), 92,77$ & 106 & $\mathrm{w}, \mathrm{x}, \mathrm{y}, \mathrm{z}$ \\
\hline$p$-Xylene ${ }^{\mathrm{b}}$ & $106-42-3$ & 865 & 862 & $91,106(59), 105(27), 77,51$ & 106 & $\mathrm{w}, \mathrm{x}, \mathrm{y}, \mathrm{z}$ \\
\hline$o$-Xylene $\mathrm{b}^{\mathrm{b}}$ & $95-47-6$ & 889 & 879 & $91,106(54), 105(21), 77,92$ & 106 & $\mathrm{w}, \mathrm{x}, \mathrm{y}, \mathrm{z}$ \\
\hline$n$-Nonane & $111-84-2$ & 900 & 900 & $43,57(94), 41(52), 85,71$ & 128 & w \\
\hline Benzene, 1-methylethyl- & $98-82-8$ & 922 & 914 & $105,120(28), 77(20), 103$ & 120 & $\mathrm{w}$ \\
\hline$\alpha$-Thujene & $2867-05-2$ & 929 & 928 & $93,91(49), 79,51$ & 136 & $\mathrm{x}$ \\
\hline p-Menthene, trans- ${ }^{\mathrm{a}}$ & $1124-25-0$ & 945 & - & $81,68(96), 95,91$ & 138 & $\mathrm{w}$ \\
\hline Benzene, propyl- & $103-65-1$ & 950 & 944 & $91,120(28), 92(12), 65,78$ & 120 & $\mathrm{w}, \mathrm{x}, \mathrm{y}, \mathrm{z}$ \\
\hline Benzene, 1-ethyl-3-methyl & $620-14-4$ & 958 & 960 & $105,120(35), 91(10), 77,106$ & 120 & $\mathrm{w}, \mathrm{x}, \mathrm{y}, \mathrm{z}$ \\
\hline Nonane, 5-methyl- & $15869-85-9$ & 959 & 961 & $43,85(71), 53(46), 84,29$ & 142 & $\mathrm{w}$ \\
\hline Benzene, 1-ethyl-4-methyl- & $622-96-8$ & 960 & 963 & $105,120(33), 91(10), 77,106$ & 120 & $\mathrm{w}, \mathrm{x}, \mathrm{y}$ \\
\hline Nonane, 4-methyl- & $52896-95-4$ & 961 & 963 & $57,43(66), 28(50), 70,71$ & 142 & $\mathrm{w}$ \\
\hline Dimethyl trisulfide & $3658-80-8$ & 962 & 976 & $126,79(41), 128(32), 64,111$ & 126 & $\mathrm{w}, \mathrm{x}, \mathrm{y}, \mathrm{z}$ \\
\hline $\begin{array}{l}\text { Aliphatic-aromatic } \\
\text { hydrocarbon }\end{array}$ & - & 965 & - & $105,120(57), 119(15), 77,91$ & 120 & $\mathrm{w}, \mathrm{x}$ \\
\hline Benzene, 1-ethyl-4-methyl- & $622-96-8$ & 966 & 963 & $105,120(56), 77(13), 91,119$ & 120 & $\mathrm{z}$ \\
\hline 1-Heptanol & $111-70-6$ & 969 & 970 & $70,55(83), 69,41$ & - & $\mathrm{w}$ \\
\hline Nonane, 3-methyl- & $5911-04-6$ & 970 & 972 & $57,71(72), 41(61), 93,43$ & - & $\mathrm{w}$ \\
\hline Sabinene & $3387-41-5$ & 971 & 976 & $93,91(50), 79(49), 69$ & 136 & $\mathrm{x}$ \\
\hline 4-Octanone & $589-63-9$ & 975 & 970 & $71,85(77), 57(57), 43,41$ & 128 & $\mathrm{x}$ \\
\hline Benzene, 1-ethyl-2-methyl- & $611-14-3$ & 976 & 976 & $105,120(34), 91(10), 77,106$ & 120 & $\mathrm{w}, \mathrm{x}, \mathrm{y}, \mathrm{z}$ \\
\hline$p$-Menthane, trans- & $1678-82-6$ & 979 & 978 & $97,55(81), 41(40), 207,69$ & 140 & w \\
\hline Benzene, 1,3,5-trimethyl- & $108-67-8$ & 989 & 994 & $105,120(52), 119(13), 77,91$ & 120 & $\mathrm{w}, \mathrm{x}, \mathrm{y}, \mathrm{z}$ \\
\hline Phenol $^{\mathrm{c}}$ & $108-95-2$ & 994 & 982 & $94,66(26), 65(21), 39,95$ & 94 & $\mathrm{w}, \mathrm{x}, \mathrm{y}, \mathrm{z}$ \\
\hline
\end{tabular}


Table 1 (continued)

\begin{tabular}{|c|c|c|c|c|c|c|}
\hline Compound & CAS\# & $L T P R I^{\operatorname{Exp}}$ & LTPRI $I^{\text {Lit }}$ & $m / z$ & $\mathrm{M}^{\bullet+}$ & Occurrence \\
\hline$n$-Decane & $124-18-5$ & 1000 & 1000 & $57,43(76), 71(45), 105,41$ & 142 & $\mathrm{w}, \mathrm{x}, \mathrm{y}, \mathrm{z}$ \\
\hline Benzene, 2-methylpropyl- & $538-93-2$ & 1003 & 1009 & $91,92,134$ & 134 & $\mathrm{x}$ \\
\hline$\alpha$-Phellandrene & $99-83-2$ & 1005 & 1005 & $93,77(51), 92(48), 85,69$ & 136 & $\mathrm{w}$ \\
\hline 3-Carene & $13466-78-9$ & 1006 & 1010 & $93,91(40), 105(37), 79,77$ & 136 & $\mathrm{w}, \mathrm{x}$ \\
\hline Benzene, 1,3-dichloro-b,c & $541-73-1$ & 1007 & 1011 & $146,148(96), 111(67), 50,281$ & 146 & $\mathrm{w}$ \\
\hline Benzene, 1,2,3-trimethyl- & $526-73-8$ & 1017 & 1020 & $105,120(48), 119(13), 77,106$ & 120 & $\mathrm{w}, \mathrm{x}, \mathrm{y}, \mathrm{z}$ \\
\hline $\begin{array}{l}\text { Benzene, } \\
\text { (1-methylethyl)-1-methyl-4- }\end{array}$ & $99-87-6$ & 1020 & 1020 & $119,134(34), 91(16), 117$ & 134 & $\mathrm{w}, \mathrm{x}, \mathrm{y}, \mathrm{z}$ \\
\hline $\begin{array}{l}\text { Benzene, } \\
\text { (1-methylethyl)-1-methyl-2- }\end{array}$ & $527-84-4$ & 1022 & 1022 & $119,134(29), 91(18), 117,120$ & 134 & w \\
\hline Nonane, 2,6-dimethyl- & $17302-28-2$ & 1022 & 1030 & $71,43(71), 57(68), 70,41$ & - & $\mathrm{w}, \mathrm{x}, \mathrm{y}, \mathrm{z}$ \\
\hline Limonene & $138-86-3$ & 1025 & 1031 & $68,93(85), 67(76), 79,94$ & 136 & $\mathrm{w}, \mathrm{x}, \mathrm{y}$ \\
\hline Cyclohexane, butyl- & $1678-93-9$ & 1027 & 1025 & $83,82(74), 55(68), 105,41$ & 140 & $\mathrm{w}, \mathrm{x}, \mathrm{y}$ \\
\hline Indane & $496-11-7$ & 1029 & 1034 & $117,118(53), 115(30), 119,91$ & 118 & $\mathrm{w}, \mathrm{x}, \mathrm{y}, \mathrm{z}$ \\
\hline Cyclopentane, pentyl- & $3741-00-2$ & 1033 & 1032 & $69,68(79), 41(63), 55,70$ & 140 & $\mathrm{w}, \mathrm{x}$ \\
\hline $\begin{array}{l}\text { Naphthalene, decahydro-, } \\
\text { trans- }\end{array}$ & $493-02-7$ & 1046 & 1057 & $138,67(73), 82(59), 95,81$ & 138 & $\mathrm{w}, \mathrm{x}, \mathrm{y}, \mathrm{z}$ \\
\hline Benzene, 1,3-diethyl- & $141-93-5$ & 1047 & 1035 & $119,105(99), 134(48), 91,103$ & 134 & $\mathrm{w}, \mathrm{x}, \mathrm{z}$ \\
\hline Benzene, 1-methyl-4-propyl- & $1074-55-1$ & 1048 & 1038 & $105,134(18), 79(11), 106,77$ & 134 & $\mathrm{w}, \mathrm{x}, \mathrm{z}$ \\
\hline Benzene, 1-methyl-3-propyl- & $1074-43-7$ & 1049 & 1055 & $105,134(29), 106(14) 91,77$ & 134 & $\mathrm{w}, \mathrm{x}, \mathrm{y}, \mathrm{z}$ \\
\hline Benzene, 1,2-diethyl- & $135-01-3$ & 1052 & 1057 & $105,134(29), 28(20), 119,91$ & 134 & $\mathrm{w}, \mathrm{x}$ \\
\hline Benzene, butyl- & $104-51-8$ & 1053 & 1054 & $91,92(68), 134(49), 119,41$ & 134 & $\mathrm{w}, \mathrm{x}, \mathrm{y}, \mathrm{z}$ \\
\hline Benzene, 1-ethyl-1,3-dimethyl- & $934-74-7$ & 1056 & 1055 & $119,134(38), 91(14), 105,120$ & 134 & $\mathrm{w}, \mathrm{x}, \mathrm{y}, \mathrm{z}$ \\
\hline Decane, 5-methyl- & $13151-35-4$ & 1058 & 1058 & $57,43(57), 85(39), 119,41$ & - & $\mathrm{w}, \mathrm{x}, \mathrm{y}$ \\
\hline Decane, 4-methyl- & $2847-72-5$ & 1061 & 1060 & $71,57(63), 43(54), 70,41$ & - & $\mathrm{w}, \mathrm{x}, \mathrm{y}, \mathrm{z}$ \\
\hline Benzene, 1-methyl-2-propyl- & $1074-17-5$ & 1063 & 1063 & $105,134(25), 77(11), 106,79$ & 134 & $\mathrm{w}, \mathrm{x}, \mathrm{y}, \mathrm{z}$ \\
\hline Decane, 2-methyl- & $6975-98-0$ & 1065 & 1064 & $57,43(89), 71(77), 85,41$ & - & $\mathrm{w}, \mathrm{x}, \mathrm{y}, \mathrm{z}$ \\
\hline Decane, 3-methyl- & $13151-34-3$ & 1071 & 1072 & $71,57(98), 85(62), 43,41$ & - & $\mathrm{w}, \mathrm{x}, \mathrm{y}, \mathrm{z}$ \\
\hline Benzene, 2-ethyl-1,4-dimethyl- & $1758-88-9$ & 1074 & 1072 & $119,134(35), 91(14), 117,115$ & 134 & $\mathrm{w}, \mathrm{x}, \mathrm{y}, \mathrm{z}$ \\
\hline Benzene, 1-ethyl-2,4-dimethyl- & 874-41-9 & 1075 & 1075 & $119,134(33), 117(14), 91,120$ & 134 & $\mathrm{w}, \mathrm{x}, \mathrm{y}, \mathrm{z}$ \\
\hline $\begin{array}{l}\text { Aliphatic cyclohexane } \\
\text { derivative }\end{array}$ & - & 1080 & - & $97,55(45), 96(38), 154,117$ & - & $\mathrm{w}$ \\
\hline Benzene, 4-ethyl-1,2-dimethyl- & $934-80-5$ & 1082 & 1088 & $119,134(30), 91(13), 120,41$ & 134 & $\mathrm{Z}$ \\
\hline Benzene, 1,2,3,4-tetramethyl- & $488-23-3$ & 1082 & 1078 & $119,134(35), 91(14), 120,117$ & 134 & $\mathrm{w}, \mathrm{x}, \mathrm{y}$ \\
\hline Phenol, 3-methyl- & $108-39-4$ & 1083 & 1075 & $108,108(94), 79(17), 77,53$ & 108 & $\mathrm{y}, \mathrm{z}$ \\
\hline Fenchone & $1195-79-5$ & 1084 & 1089 & $81,69(47), 41$ & 152 & $\mathrm{x}$ \\
\hline Isoterpinolene & $586-63-0$ & 1085 & 1086 & $136,91(99), 41(67), 111,83$ & 136 & $\mathrm{x}$ \\
\hline NN & - & 1085 & - & $69,111(92), 121(68), 79,125$ & - & w \\
\hline Naphthalene, decahydro-, cis- & $493-01-6$ & 1089 & 1097 & $81,96(96), 138(79), 67,82$ & 138 & $\mathrm{x}$ \\
\hline$n$-Undecane & $1120-21-4$ & 1100 & 1100 & $57,43(73), 71(59), 85,41$ & 156 & $\mathrm{w}, \mathrm{x}, \mathrm{y}, \mathrm{z}$ \\
\hline Benzene, 1-ethyl-2,3-dimethyl- & $933-98-2$ & 1106 & 1104 & $134,119(97), 81(73), 207,77$ & 134 & $\mathrm{w}, \mathrm{y}$ \\
\hline 1,3,8-p-Menthatriene & $21195-59-5$ & 1111 & 1111 & $119,134(53), 117(15), 91,115$ & 134 & $\mathrm{w}, \mathrm{x}, \mathrm{y}, \mathrm{z}$ \\
\hline Benzene, 1,2,4,5-tetramethyl- & $95-93-2$ & 1115 & 1123 & $119,134(51), 91(11), 115,120$ & 134 & $\mathrm{w}, \mathrm{x}, \mathrm{y}, \mathrm{z}$ \\
\hline $\begin{array}{l}\text { Naphthalene, } \\
\text { decahydro- } \alpha \text {-methyl-, trans- }{ }^{\mathrm{a}}\end{array}$ & $2547-27-5$ & 1117 & - & $152,82(86), 95(84), 84,127$ & 152 & $\mathrm{w}, \mathrm{x}, \mathrm{y}, \mathrm{z}$ \\
\hline Decane, 2,6-dimethyl- & $13150-81-7$ & 1121 & 1119 & 57, 71(79), 119(59), 112, 109 & 170 & w \\
\hline Branched alkane & - & 1123 & - & $71,57(87), 70(62), 43,56$ & - & $\mathrm{w}$ \\
\hline Decane, 3,7-dimethyl- & $17312-54-8$ & 1127 & 1125 & $71,57(99), 85(84), 43,70$ & - & $\mathrm{w}, \mathrm{x}, \mathrm{y}, \mathrm{z}$ \\
\hline Cyclohexane, pentyl- & $4292-92-6$ & 1130 & 1130 & $83,82(75), 55(62), 69,41$ & 154 & $\mathrm{w}, \mathrm{x}, \mathrm{y}, \mathrm{z}$ \\
\hline Decane, 3,8-dimethyl- & $17312-55-9$ & 1133 & 1140 & $57,71(71), 43(46), 85,41$ & - & $\mathrm{y}$ \\
\hline Cyclopentane, hexyl- & $4457-00-5$ & 1136 & 1134 & $69,41(99), 68(94), 83,84$ & 154 & $\mathrm{w}, \mathrm{y}, \mathrm{z}$ \\
\hline Cyclohexanone, 2-propyl- & $94-65-5$ & 1136 & 1134 & $98,55(25), 32,41$ & 140 & $\mathrm{x}, \mathrm{z}$ \\
\hline
\end{tabular}


Table 1 (continued)

\begin{tabular}{|c|c|c|c|c|c|c|}
\hline Compound & CAS\# & $L T P R I^{E x p}$ & LTPRI $I^{\text {Lit }}$ & $m / z$ & $\mathrm{M}^{\bullet+}$ & Occurrence \\
\hline $\begin{array}{l}\text { Naphthalene, } \\
\text { decahydro-2-methyl- }\end{array}$ & $2958-76-1$ & 1140 & 1138 & $57,95(83), 85(80), 111,152$ & 152 & $\mathrm{w}, \mathrm{x}, \mathrm{y}$ \\
\hline Benzene, 1,2,3,5-tetramethyl- & $527-53-7$ & 1147 & 1145 & $119,134(61), 41(43), 81,91$ & 134 & $\mathrm{w}, \mathrm{x}, \mathrm{y}$ \\
\hline$p$-Menthone & $89-80-5$ & 1150 & 1154 & $112,71(73), 73(68), 69,105$ & 154 & $\mathrm{w}, \mathrm{x}, \mathrm{z}$ \\
\hline Undecane, 6-methyl- & $17302-33-9$ & 1155 & 1154 & $57,98(35), 43(22), 41,56$ & - & $\mathrm{w}, \mathrm{x}, \mathrm{y}$ \\
\hline $\begin{array}{l}\text { Naphthalene, } 1,2,3 \text {, } \\
\text { 4-tetrahydro- }\end{array}$ & $119-64-2$ & 1155 & 1158 & $104,98(91), 57(70), 132,58$ & 132 & $\mathrm{w}$ \\
\hline Undecane, 5-methyl- & $1632-70-8$ & 1156 & 1156 & $71,43(98), 85(68), 84,112$ & - & $\mathrm{w}, \mathrm{x}, \mathrm{y}, \mathrm{z}$ \\
\hline Undecane, 4-methyl- & 2980-69-0 & 1160 & 1160 & $71,43(74), 85(48), 57,70$ & - & $\mathrm{w}, \mathrm{x}, \mathrm{y}, \mathrm{z}$ \\
\hline Undecane, 2-methyl- & $7045-71-8$ & 1167 & 1164 & $43,57(98), 71(81), 85,41$ & - & $\mathrm{w}$ \\
\hline Undecane, 3-methyl- & $1002-43-3$ & 1171 & 1171 & $57,85(64), 71(62), 43,41$ & - & $\mathrm{w}, \mathrm{x}, \mathrm{y}, \mathrm{z}$ \\
\hline Menthol & $89-78-1$ & 1170 & 1173 & $71,57(57), 41(49), 43,86$ & - & $\mathrm{y}, \mathrm{z}$ \\
\hline Naphthalene $e^{c}$ & $91-20-3$ & 1176 & 1179 & $128,127(16), 28(12), 95,129$ & 128 & $\mathrm{w}, \mathrm{x}, \mathrm{y}, \mathrm{z}$ \\
\hline Cyclohexane derivative & - & 1182 & - & $97,55(53), 131(20), 96$ & - & $\mathrm{w}, \mathrm{x}, \mathrm{y}$ \\
\hline $\mathrm{NN}$ & - & 1186 & - & $69,83(96), 91(89), 168,111$ & - & $\mathrm{w}, \mathrm{x}, \mathrm{y}$ \\
\hline $\mathrm{NN}$ & - & 1196 & - & $97,133(68), 96(66), 95$ & - & $\mathrm{w}, \mathrm{x}$ \\
\hline$n$-Dodecane & $112-40-3$ & 1200 & 1200 & $57,43(73), 71(64), 85,41$ & 170 & $\mathrm{w}, \mathrm{x}$ \\
\hline Undecane, 2,6-dimethyl- & $17301-23-4$ & 1214 & 1216 & $57,71(48), 43(34), 41,56$ & 184 & $\mathrm{w}, \mathrm{x}, \mathrm{y}, \mathrm{z}$ \\
\hline $\begin{array}{l}\text { Naphthalene, } \\
\text { 2-ethyldecahydro- }\end{array}$ & $66660-40-0$ & 1216 & - & $81,137(83), 95(78), 97,109$ & 166 & $\mathrm{w}, \mathrm{x}, \mathrm{y}, \mathrm{z}$ \\
\hline Undecane, 3,7-dimethyl- & 17301-29-0 & 1221 & 1222 & $43,57(98), 85(79), 71,41$ & - & $\mathrm{w}, \mathrm{x}$ \\
\hline Cyclohexane, hexyl- & $4292-75-5$ & 1234 & 1233 & $83,82(84), 55(57), 67,41$ & 168 & $\mathrm{w}, \mathrm{x}, \mathrm{y}, \mathrm{z}$ \\
\hline Caprolactam $^{\mathrm{a}}$ & $105-60-2$ & 1244 & - & $55,113(64), 84,85$ & 113 & $\mathrm{x}$ \\
\hline Dodecane, 6-methyl- & $6044-71-9$ & 1253 & 1253 & $57,71(67), 43(43), 41,29$ & - & $\mathrm{w}, \mathrm{x}, \mathrm{y}$ \\
\hline Dodecane, 5-methyl- & 17453-93-9 & 1255 & 1255 & $43,85(89), 84(68), 83,97$ & - & $\mathrm{w}, \mathrm{x}, \mathrm{y}$ \\
\hline Dodecane, 4-methyl- & 6117-97-1 & 1259 & 1260 & $71,43(72), 85(65), 70,57$ & - & $\mathrm{w}, \mathrm{x}, \mathrm{y}$ \\
\hline Dodecane, 2-methyl- & $1560-97-0$ & 1263 & 1265 & $57,43(82), 85(62), 70,71$ & - & $\mathrm{w}, \mathrm{x}, \mathrm{y}$ \\
\hline Dodecane, 3-methyl- & $17312-57-1$ & 1270 & 1271 & $57,85(55), 43,71$ & - & $\mathrm{w}$ \\
\hline Decane, 1-chloro- & $1002-69-3$ & 1264 & 1261 & $43,85(88), 57(88), 71,69$ & - & $\mathrm{w}, \mathrm{y}$ \\
\hline Dodecane, 3-methyl- & $17312-57-1$ & 1273 & 1271 & $71,57(99), 43(45), 113,112$ & - & $\mathrm{w}, \mathrm{x}, \mathrm{y}$ \\
\hline Naphthalene, 2-methyl- & $91-57-6$ & 1286 & 1291 & $141,142(85), 71(47), 115,83$ & 142 & $\mathrm{w}, \mathrm{x}, \mathrm{y}, \mathrm{z}$ \\
\hline Indole & $120-72-9$ & 1294 & 1292 & $117,90(38), 89(26), 118,116$ & 117 & $\mathrm{y}, \mathrm{z}$ \\
\hline$n$-Tridecane & $629-50-5$ & 1300 & 1300 & $57,43(77), 71(72), 85,41$ & 184 & $\mathrm{w}, \mathrm{x}, \mathrm{y}, \mathrm{z}$ \\
\hline Naphthalene, 1-methyl- & $90-12-0$ & 1301 & 1308 & $142,141(85), 115,117$ & 142 & $\mathrm{x}, \mathrm{z}$ \\
\hline $\begin{array}{l}\text { Naphthalene, 1,2,3, } \\
\text { 4-tetrahydro-2,7-dimethyl- }\end{array}$ & $13065-07-1$ & 1311 & 1302 & $160,117(49), 118,71$ & 160 & $\mathrm{x}, \mathrm{z}$ \\
\hline Phthalic anhydride & $85-44-9$ & 1313 & 1319 & $104,76(57), 50(25), 74,148$ & 148 & w \\
\hline $\begin{array}{l}\text { Nonane, } 2,2,4,4,6,8, \\
\text { 8-heptamethyl- }\end{array}$ & $4390-04-9$ & 1317 & 1323 & $57,41(18), 85(18), 71,99$ & - & $\mathrm{w}, \mathrm{x}$ \\
\hline Tridecane, 7-methyl- & $26730-14-3$ & 1351 & 1351 & $57,71(81), 159(50), 105$ & - & $\mathrm{x}$ \\
\hline Tridecane, 4-methyl- & $26730-12-1$ & 1359 & 1360 & $57,71(88), 43(66), 85$ & - & $\mathrm{x}$ \\
\hline Tridecane, 2-methyl- & $1560-96-9$ & 1363 & 1365 & $85,57(71), 71(67), 97,43$ & - & $\mathrm{x}$ \\
\hline Tridecane, 3-methyl- & $6418-41-3$ & 1370 & 1371 & $57,71(62), 28(50), 43,41$ & - & $\mathrm{x}, \mathrm{z}$ \\
\hline Copaene & $3856-25-5$ & 1370 & 1376 & $105,119(97), 161(63), 81,77$ & 204 & $\mathrm{y}$ \\
\hline Dodecane, 2,6,10-trimethyl- & $3891-98-3$ & 1376 & 1382 & $57,71(88), 85(56), 41,43$ & - & $\mathrm{w}, \mathrm{x}$ \\
\hline Diphenyl ether ${ }^{\mathrm{a}}$ & $101-84-8$ & 1396 & - & $170,141(62), 142(30), 77,168$ & 170 & $\mathrm{x}, \mathrm{y}, \mathrm{z}$ \\
\hline$\beta$-Elemene & $515-13-9$ & 1397 & 1391 & $189,93(92), 161(85), 65,137$ & 204 & $\mathrm{w}$ \\
\hline$n$-Tetradecane & $629-59-4$ & 1400 & 1400 & $57,43(62), 71(59), 85,41$ & 198 & $\mathrm{w}, \mathrm{x}, \mathrm{y}, \mathrm{z}$ \\
\hline (E) $\beta$-Caryophyllene & $87-44-5$ & 1413 & 1419 & $91,133(93), 93(93), 79$ & 204 & $\mathrm{w}, \mathrm{x}, \mathrm{y}$ \\
\hline Branched alkane & - & 1417 & - & $57,71(91), 85(72), 43,70$ & - & $\mathrm{w}, \mathrm{x}, \mathrm{z}$ \\
\hline Branched alkane & - & 1423 & - & $57,71(71), 97(69), 55,56$ & - & $\mathrm{w}, \mathrm{x}, \mathrm{y}, \mathrm{z}$ \\
\hline Branched alkane & - & 1439 & - & $57,71(86), 113(46), 43,85$ & - & $\mathrm{w}, \mathrm{x}, \mathrm{y}, \mathrm{z}$ \\
\hline Branched alkane & - & 1441 & - & $57,71(72), 43(55), 112,197$ & - & $\mathrm{w}, \mathrm{x}, \mathrm{z}$ \\
\hline
\end{tabular}


Table 1 (continued)

\begin{tabular}{|c|c|c|c|c|c|c|}
\hline Compound & CAS\# & $L T P R I^{\operatorname{Exp}}$ & $L T P R I^{\text {Lit }}$ & $m / z$ & $\mathrm{M}^{\bullet+}$ & Occurrence \\
\hline$\overline{\alpha-\text { Humulene }}$ & $6753-98-6$ & 1448 & 1453 & $93,71(46), 57(40), 80$ & 204 & $\mathrm{w}, \mathrm{x}, \mathrm{y}$ \\
\hline Tetradecane, 5-methyl- & $25117-24-2$ & 1454 & 1454 & $57,85(76), 169$ & 212 & $\mathrm{w}, \mathrm{x}, \mathrm{y}$ \\
\hline Tetradecane, 2-methyl- & $1560-95-8$ & 1461 & 1465 & $57,85(78), 97(56), 55,83$ & 212 & $\mathrm{w}, \mathrm{x}, \mathrm{y}$ \\
\hline Tetradecane, 3-methyl- & $18435-22-8$ & 1468 & 1472 & $57,71(79), 85(62), 113,70$ & - & $\mathrm{w}$ \\
\hline Acenaphthene & $83-32-9$ & 1472 & 1476 & $154,153(98), 125,70$ & 154 & $\mathrm{x}, \mathrm{z}$ \\
\hline Branched alkane & - & 1477 & - & $71,57(92), 43(61), 113,70$ & - & $\mathrm{w}, \mathrm{x}, \mathrm{y}, \mathrm{z}$ \\
\hline$n$-Pentadecane & $629-62-9$ & 1499 & 1500 & $57,71(77), 85(59), 43,41$ & 212 & $\mathrm{w}, \mathrm{x}, \mathrm{y}, \mathrm{z}$ \\
\hline Butylated hydroxytoluene & $128-37-0$ & 1511 & 1512 & $205,220(27), 206(180), 145,177$ & 220 & $\mathrm{w}, \mathrm{x}, \mathrm{y}$ \\
\hline Calamene & $483-77-2$ & 1519 & 1523 & $159,160(15), 128(13), 202,43$ & 202 & $\mathrm{y}$ \\
\hline Benzene, 1-butylhexyl- $^{\mathrm{a}}$ & $4537-11-05$ & 1535 & - & $91,147(31), 161(20), 104,29$ & 218 & $\mathrm{w}, \mathrm{z}$ \\
\hline Benzene, 1,3,5-tri-tert-butyl- ${ }^{\mathrm{a}}$ & $1460-02-2$ & 1555 & - & $231,246(28), 216(24), 232,215$ & 246 & $\mathrm{w}$ \\
\hline Pentadecane, 2-methyl- & $1560-93-6$ & 1569 & 1564 & $57,71(59), 43,55$ & 226 & $\mathrm{z}$ \\
\hline Diethyl phthalate ${ }^{c}$ & $84-66-2$ & 1594 & 1591 & $149,177(23), 32,28$ & - & $\mathrm{w}$ \\
\hline$n$-Hexadecane & $544-76-3$ & 1599 & 1600 & $57,71(61), 85(50), 43,55$ & 226 & $\mathrm{w}, \mathrm{x}, \mathrm{z}$ \\
\hline Benzophenone $^{c}$ & 119-61-9 & 1623 & 1640 & $105,182(48), 77$ & 182 & $\mathrm{w}, \mathrm{x}$ \\
\hline Benzene, 1-butylheptyl- & $4537-15-9$ & 1633 & 1628 & $91,44(43), 105(37), 147$ & 232 & $\mathrm{w}, \mathrm{z}$ \\
\hline Benzene, 1-propyloctyl- & $4536-86-1$ & 1643 & 1638 & $91,92(41), 43,189$ & 232 & w \\
\hline $\begin{array}{l}\text { Naphthalene, 1, } \\
\text { 6-dimethyl-4-(1-methylethyl)- }\end{array}$ & $483-78-3$ & 1671 & 1674 & $183,198(63)$ & 198 & w \\
\hline Benzene, 1-pentylheptyl- & $2719-62-2$ & 1727 & 1725 & $91,105(27), 191,39$ & 246 & $\mathrm{z}$ \\
\hline Benzene, 1-butyloctyl- & $2719-63-3$ & 1731 & 1729 & $91,71(46), 85(32), 57,69$ & 246 & $\mathrm{z}$ \\
\hline Isopropyl myristate & $110-27-0$ & 1818 & 1827 & $43,228(72), 57(67), 102,41$ & - & $\mathrm{w}$ \\
\hline Dibutyl phthalate $^{\mathrm{c}}$ & $84-74-2$ & 1960 & 1954 & $149,150(11), 223(6), 104,41$ & - & $\mathrm{w}, \mathrm{y}$ \\
\hline Cyclic octaatomic sulfur & $10544-50-0$ & 1998 & 2004 & $64,256(59), 160(48), 192,128$ & 256 & $\mathrm{x}, \mathrm{z}$ \\
\hline Diisooctyl phthalate $^{c}$ & $27554-26-3$ & 2545 & 2540 & $149,167(33), 57(16), 279,150$ & - & $\mathrm{w}, \mathrm{x}$ \\
\hline$n$-Heptacosane & $593-49-7$ & 2699 & 2700 & $57,71(81), 43(64), 85,41$ & 380 & $\mathrm{x}$ \\
\hline n-Octacosane & $630-02-4$ & 2803 & 2800 & $57,71(72), 43(69), 85,55$ & 394 & $\mathrm{x}$ \\
\hline$n$-Nonacosane & $630-03-5$ & 2903 & 2900 & $57,71(83), 43(56), 85,55$ & 408 & $\mathrm{x}$ \\
\hline$n$-Triacontane & $638-68-6$ & 2999 & 3000 & $57,71(82), 85(64), 43,55$ & 422 & $\mathrm{x}$ \\
\hline$n$-Hentriaconatane & $630-04-6$ & 3100 & 3100 & $57,71(75), 43(52), 85,97$ & 436 & $\mathrm{x}$ \\
\hline$n$-Dotriacontane & $544-85-4$ & 3198 & 3200 & $57,71(67), 43(56), 85,55$ & 450 & $\mathrm{x}$ \\
\hline$n$-Tritriacontane & $630-05-7$ & 3302 & 3300 & $57,43(91), 71(82), 85$ & 462 & $\mathrm{x}$ \\
\hline
\end{tabular}

$L_{\text {LPRI }}{ }^{\text {Exp }}$ linear temperature programmed retention index determined in the course of research, LTPRI ${ }^{\text {Lit }}$ retention index found in the literature, $\mathrm{m} / \mathrm{z}$ mass/charge ratio values of main and most intensive peaks of the mass spectrum (intensities are given in parenthesis), $M^{+}$mass of the molecular ion, $w$ laboratory investigation with CAR/PDMS fiber coating, $x$ laboratory investigation with DVB/CAR/PDMS fiber coating, $y$ field investigation with CAR/PDMS fiber coating, $z$ field investigation with DVB/CAR/PDMS fiber coating

aTentatively identified compounds

${ }^{\mathrm{b}}$ Compounds included in EPA list of target compounds

${ }^{\mathrm{c} C o m p o u n d s}$ included in EPA list of priority pollutants

detected compounds) of aliphatic hydrocarbons ranged between $21 \%$ and $83 \%$ of TIC in the course of research carried out in various variants and on various dates.

Both monocyclic compounds-benzene, toluene, xylenes, and others, as well as naphthalene and its derivatives were found in the group of aromatic hydrocarbons, which constituted between $8 \%$ and $48 \%$ of TIC, whereas terpenes made from $1 \%$ to over $13 \%$ of total VOCs and SVOCs emission through sludge. The content of other groups of compounds lay within the range from less than $1 \%$ to $10 \%$ of TIC depending on the variant and time of determination. Substances containing sulfur included such compounds as carbon disulfide, dimethyl sulfide, dimethyl disulfide, and cyclic octaatomic sulfur. Among others, in the group of carbonyl compounds, the following were identified: acetone, 3-pentanone, 4octanone, whereas esters included ethyl acetates 
Fig. 1 The chromatograms obtained during HS-SPME/GC-MS analysis of sewage samples:

a laboratory investigation with CAR/PDMS fiber coating;

b laboratory investigation with DVB/CAR/PDMS fiber coating; c field investigation with CAR/PDMS fiber coating;

d field investigation with DVB/CAR/PDMS fiber coating

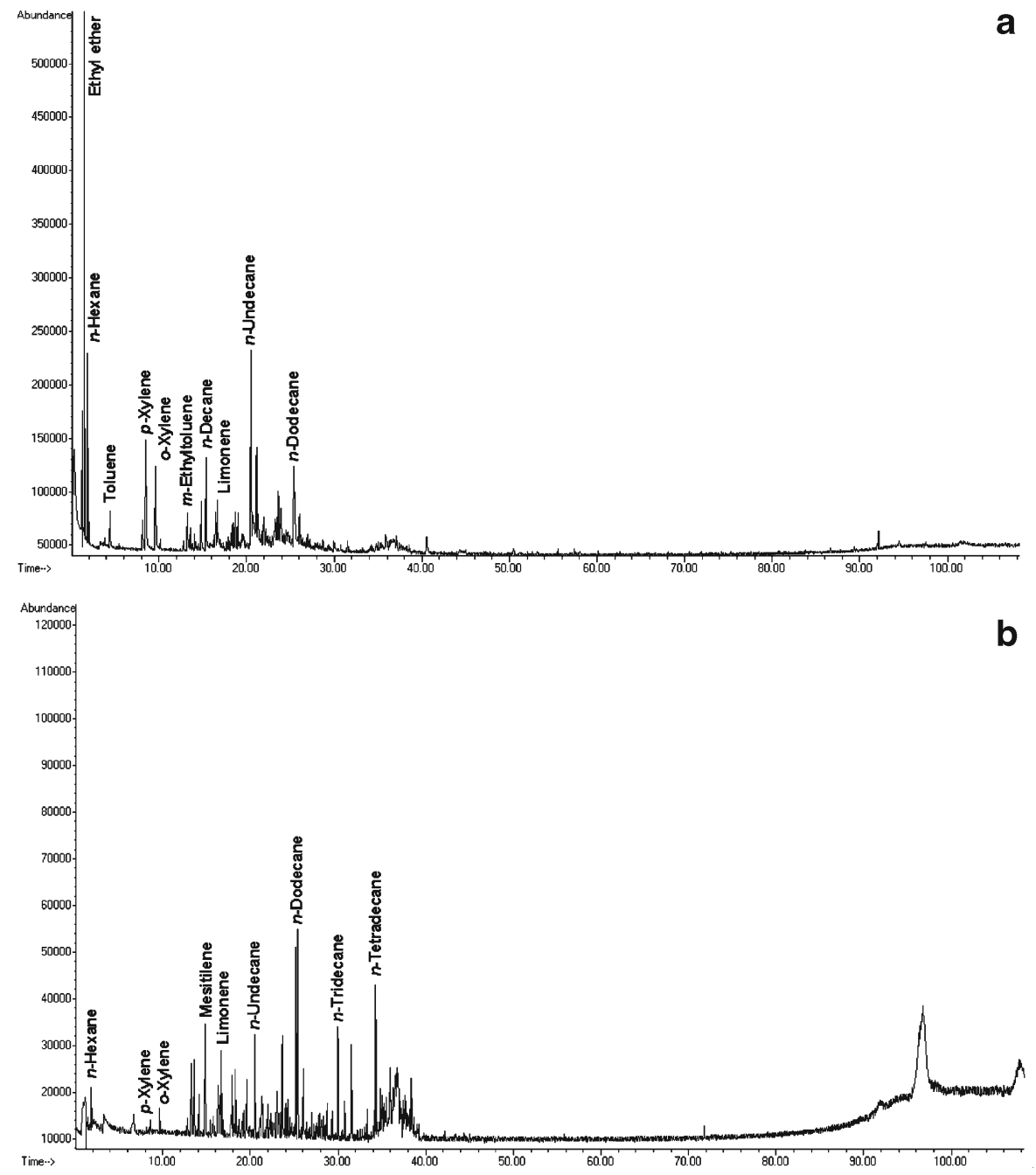

and phthalic acids. Main chlorine compounds determined in the sewage sludge were: methylene chloride, 1,2-dichloroethene, trichloroethylene, bromodichloromethane, and tetrachloroethylene. Percentages of individual groups of compounds identified in the laboratory analysis using SPME fibers with different coatings are shown in Fig. 2.

Detected and identified were also about 100 organic compounds emitted from the sewage sludge stored on the heap. A few compounds identified in the course of extraction carried out on the heap, including: triethylamine, 2-butanone, 1-ethyl-4-methylbenzene, 4-ethyl-1,2-dimethylbenzene, 3-methylphenol, menthol, indole, copaene, calamene, 2-methylpentadecane, 1-pentylheptyl- benzene, and 1-butyloctylbenzene were not recorded during laboratory determinations. Among compounds extracted directly on the sludge heap, the following compounds were characterized with greatest intensity: dimethyl disulfide, dimethyl trisulfide, mesitylene, toluene, $p$-xylene, $o$-xylene, limonene, $n$-decane, $n$-undecane, indoles, and ethylbenzene. Similarly, as during laboratory research, the largest group made aliphatic hydrocarbons of linear, branched, and cyclical structure ( $27 \%$ to $58 \%$ of total VOC emission) and aromatic hydrocarbons, i.e., benzene, naphthalene, and their derivatives (14\% to 55\%). Significant portion (5\% to over $25 \%$ ) of VOC emission through the sludge heap makes sulfur compounds, what is a substantial increase in comparison with 
Fig. 1 (continued)

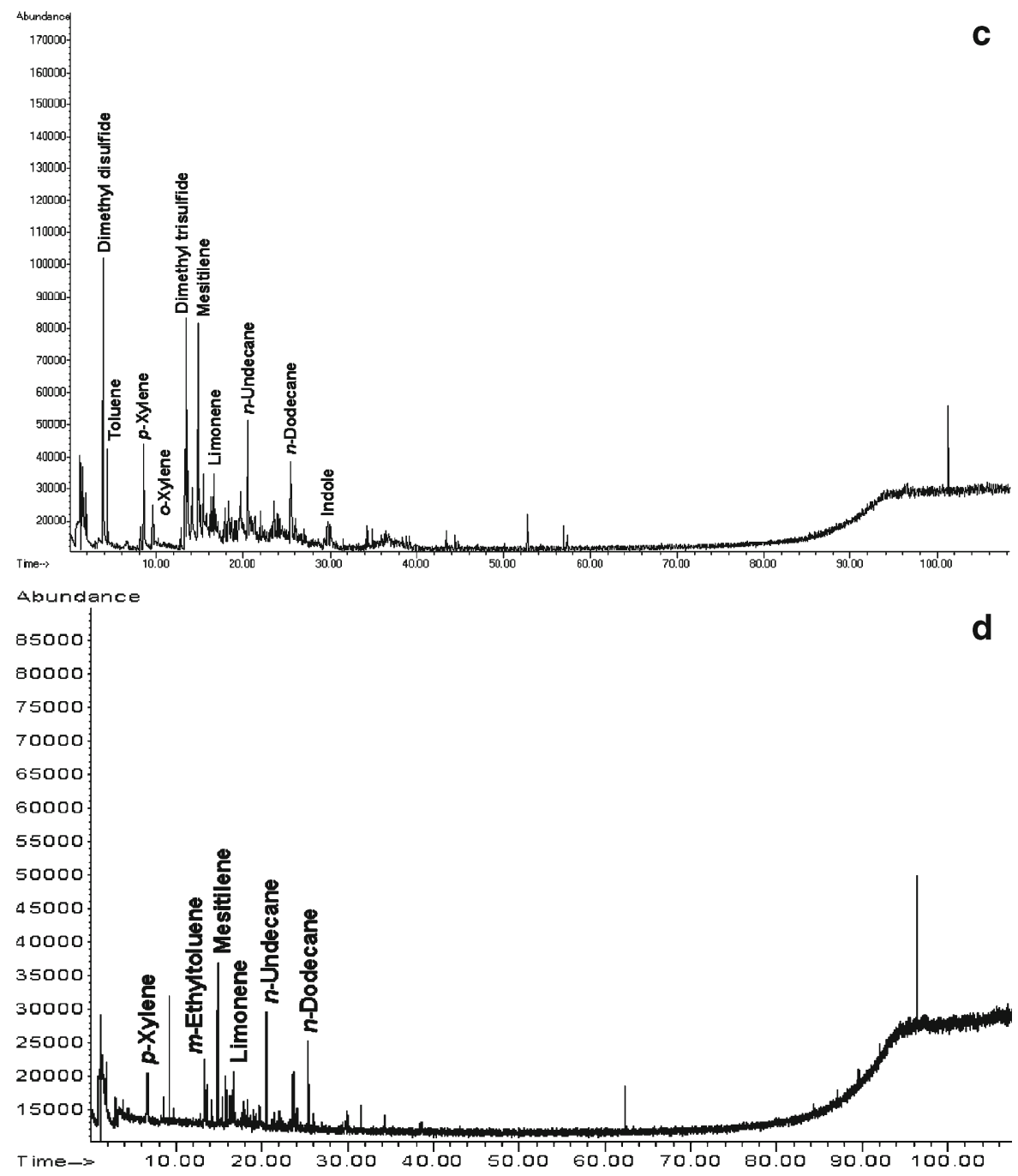

the results of laboratory research. During research on the heap were also detected: terpenes $(2.5 \%$ to $5 \%$, main compounds: limonene, menthone), nitrogen compounds (from almost $1 \%$ to $8 \%$, e.g., indole, trimethylamine), as well as ketones, alcohols, ethers, esters, and chlorine compounds, whose content varied within the range from $0 \%$ to $4 \%$. Percentage of individual groups of compounds determined in field analysis is provided in Fig. 3.

Volatile and semi-volatile organic compounds detected in the sewage sludge can be divided into two groups: compounds of natural origin and synthetic substances. The majority of compounds extracted from the sewage sludge composed of natural compounds derived from human excre- ments, nutritional remains, and products of their decomposition. Among VOCs detected in the sludge substances of natural origin were:

- Simple and branched alcanes, e.g., decane, undecane, contained in plant waxes;

- Terpenes and terpenoids, e.g., phellandrene, 3 -carene, derived from plant tissues;

- Substances of final products of various organic metabolic processes, including organisms forming active sludge, e.g., sulfane, carbon disulfide, dimethyl polysulfides, methanol, ethanol.

Synthetic substances present in the sewage sludge are less susceptible to biodegradation than natural compounds; thus, they can accumulate in 
Fig. 2 The percentage of individual group of compounds obtained in laboratory analysis with different fiber coatings. a CAR/PDMS;

b DVB/CAR/PDMS.

$A L H$ aliphatic

hydrocarbons, $A R H$

aromatic hydrocarbons,

$T E R$ terpenes, $S U L$

sulfur compounds, ETH

ethers, $A L C$ alcohols,

$C H L$ chloride

compounds, EST esters, $C A R$ carbonyls, $N I T$

nitrogen compounds, $N N I$

nonidentified compounds

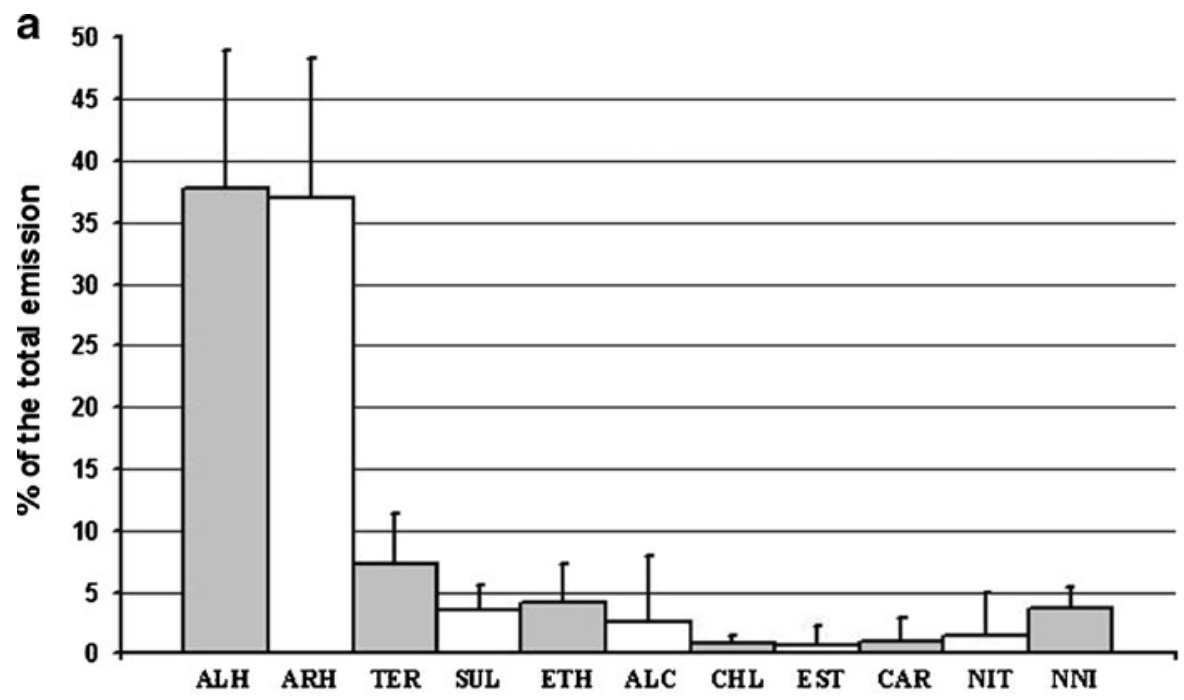

b

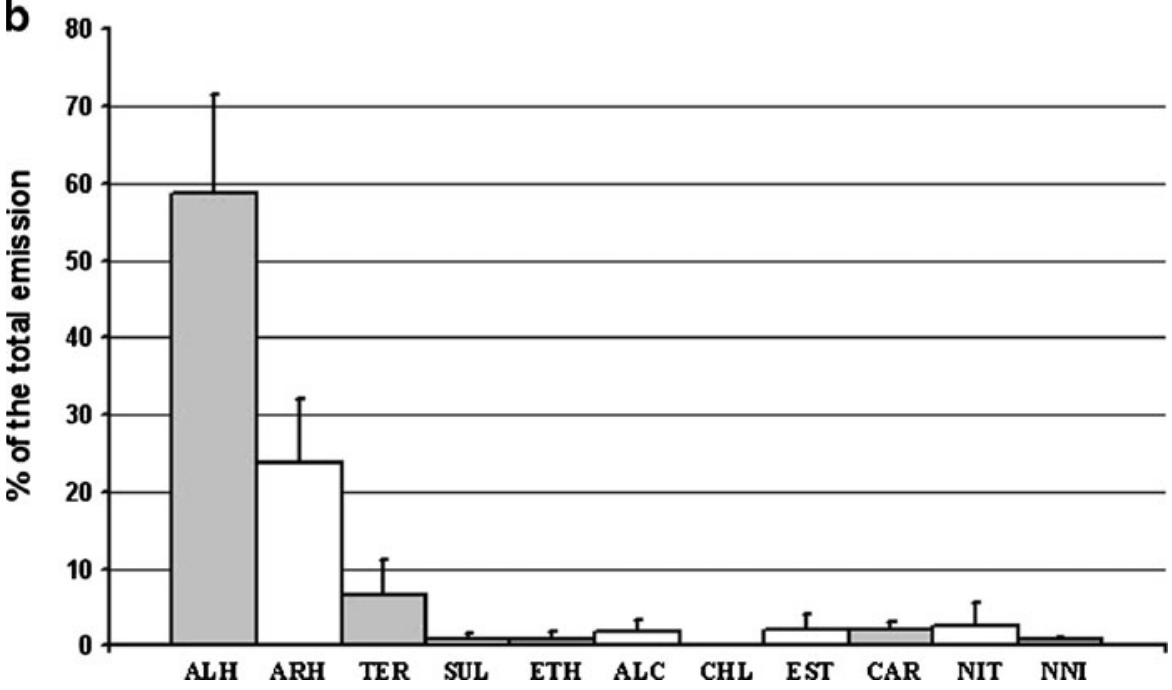

the sludge and-eventually-in the natural environment. Synthetic substances emitted from the sewage included:

- Alkylobenzenes, e.g., mesitylene, xylenescomponents of solvents, synthetic oils, greases, bitumen masses;

- Chlorine-containing substances: chloroform and methylene chloride-popular organic solvents, benzene chloride-intermediate product of pharmaceutical industry and component of dyestuffs, bromodichloromethane, and dichloroethenes-by-products of water disinfection;
- Remains of hygiene articles used in the household: limonene and menthol-aromatizing agents for washing-up liquids and toothpastes,

- Plasticizers-diethyl phthalate, diisooctyl phthalate, dibutyl phthalate, phthalic anhydride;

- Butylated hydroxytoluene-popular antioxidant added to food, cosmetics, drugs, rubber, etc.;

- Benzophenone-sunscreen agent;

- PAH, e.g., naphthalene-used as an intermediate product in the production of dyestuffs, dissolvents, synthetic resins, and insecticides.

Some substances detected in the sludge are known for their harmful influence on human 
Fig. 3 The percentage of individual group of compounds obtained in field analysis with different fiber coatings. a CAR/PDMS;

b DVB/CAR/PDMS.

$A L H$ aliphatic

hydrocarbons, $A R H$

aromatic hydrocarbons,

TER terpenes, $S U L$

sulfur compounds, $E T H$

ethers, $A L C$ alcohols,

$C H L$ chloride

compounds, EST esters,

$C A R$ carbonyls, $N I T$

nitrogen compounds, $N N I$

nonidentified compounds

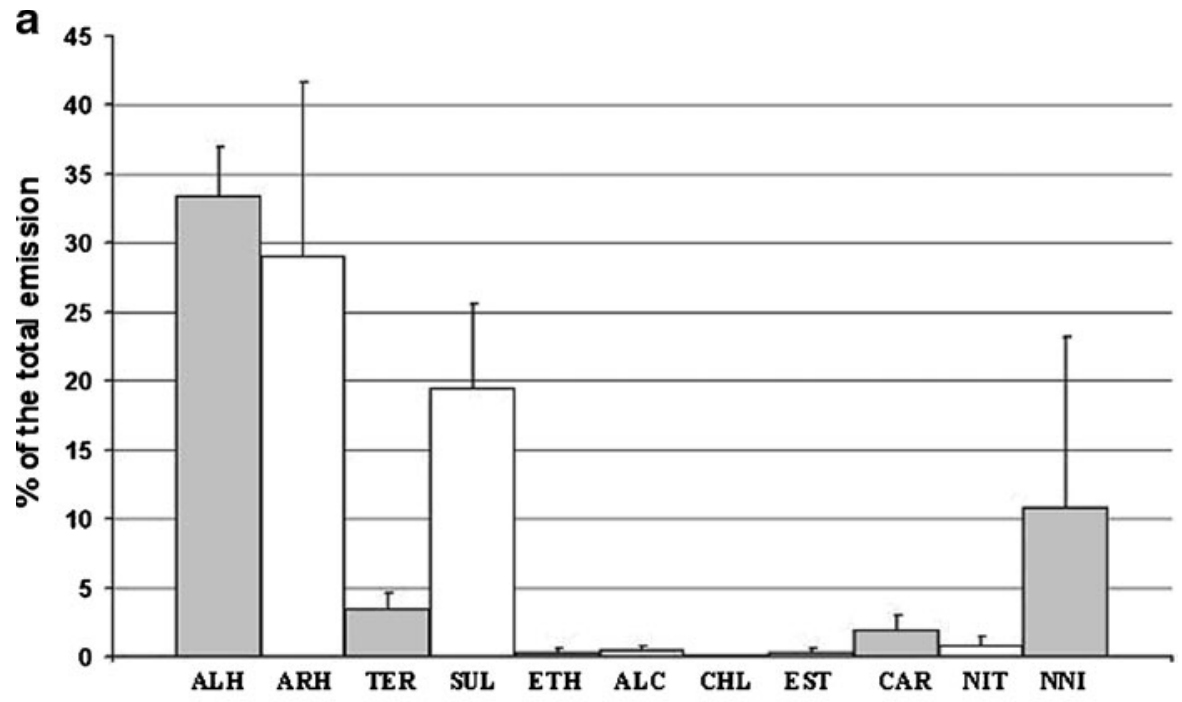

b

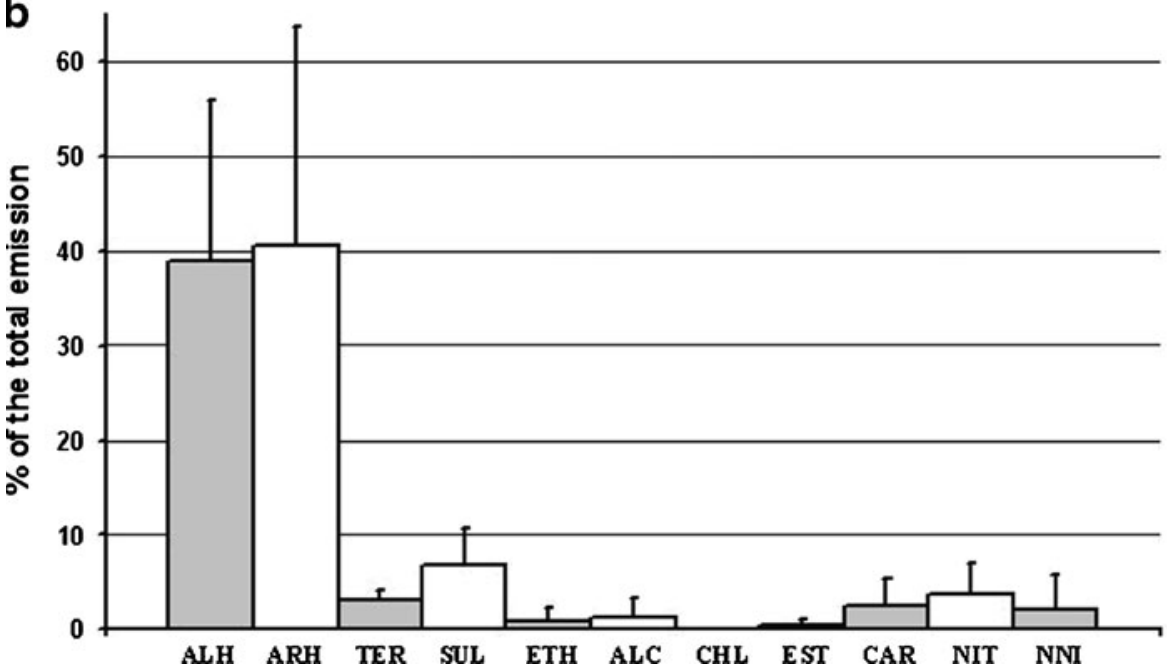

health and natural environment. Sixteen compounds (marked with superscripted "c" in Table 1) detected in the sludge are included on the list of priority pollutants established by EPA, and other 16 chemicals (marked with superscripted " $b$ " in Table 1) are on the EPA's list of target compounds.

Phthalic acid esters are counted as endocrine disrupters and they alter the normal functioning of the endocrine system and cause important reproductive and developmental alterations, such as feminization and decreased fertility (Arcadi et al. 1998). Naphthalene is defined by the US Environmental Protection Agency as hazardous to environment. Exposition to high concentration of naphthalene can damage or destroy red blood cells and cause hemolytic anemia, (Lu et al. 2005). Carbon disulfide has been classified in the US as a hazardous air pollutant in Title III of the Clean Air Act Amendments of 1990. Hydrogen sulfide is subjected to a stringent control for its environmental release due to its toxicity, unpleasant odor, and corrosive properties (Janssen et al. 1997). Benzene, toluene, ethyl benzene, and xylenes are known carcinogens and pose a risk to both human health as well as other forms of life (Fang et al. 2004). The activity of butylated hydroxytoluene has been found to be ambiguous as its toxicological allegations are still being questioned. Thus, in some studies, cancer growth related to its 
activity was shown to be increasing but decreasing in others (Tryphonas et al. 1999; Safer and al-Nughamish 1999; Parke and Lewis 1992).

\section{Conclusions}

In the study, the headspace mode of solidphase micro-extraction to extract volatile and semi-volatile organic compounds from wastewater sludge was used. The HS-SPME technique combined with GC-MS offered a possibility to isolate and detect a wide spectrum of organic pollutants starting from extremely volatile compounds (e.g., methanol, ethanol, acetone) to finish with the compounds of moderate volatility such as phthalic acid esters, hydrocarbons of more than 20 carbon atoms in the molecule. Using the SPME method, the isolation and concentration process of analytes occurs in one stage, and the risk of contamination of the sample by reagents and vessels is considerably reduced. The combination of the two independent parameters: mass spectra and retention indices, allows us to obtain high identification reliability of unknown organic compounds. However, these results are, as yet, preliminary and must be supplemented by precise quantitative analysis. In quantitative analysis, in order to improve SPME extraction results, the use of additional isolation techniques, such as sorbent tubes or traps sampling should be considered.

The presence of substances having harmful influence on human health and natural environment shows the need to monitor such compounds. The monitoring could be especially important in the process of making decisions concerning the methods of municipal sewage sludge management.

Acknowledgements The authors would like to thank Mrs. Barbara Grudzień and Management of the Wastewater Treatment Plant in Białystok for their help in sample taking.

Open Access This article is distributed under the terms of the Creative Commons Attribution Noncommercial License which permits any noncommercial use, distribution, and reproduction in any medium, provided the original author(s) and source are credited.

\section{References}

Adams, R. P. (1995). Identification of essential oil components by GC/MS. Carol Stream (IL): Allured.

Arcadi, R., Costa, C., \& Imperatore, C. (1998). Oral toxicity of DEHP during pregnancy and sucking in the long-evans rat. Food and Chemical Toxicology, 36, 963-974.

Arthur, C. L., \& Pawliszyn, J. (1990). Solid phase microextraction with thermal desorption using fused silica optical fibers. Analytical Chemistry, 62, 2145-2148.

ASTM (1998). ASTMD6169: Standard practice for selection of sorbents, sampling and thermal desorption analysis procedures for volatile organic chemicals in air. West Conshohocken: ASTM.

Basheer, C., \& Lee, H. K. (2004). Hollow fiber membraneprotected solid-phase microextraction of triazine herbicides in bovine milk and sewage sludge samples. Journal of Chromatography A, 1047, 189-194.

Blount, B. C., Kobelski, R. J., McElprang, D. O., Ashley, D. L., Morrow, J. C., Chambers, D. M., et al. (2006). Quantification of 31 volatile organic compounds in whole blood using solid-phase microextraction and gas chromatography-mass spectrometry. Journal of Chromatography B, 832, 292-301.

Bravo-Linares, C. M., Mudge, S. M., \& Loyola-Sepulveda, R. H. (2007). Occurrence of volatile organic compounds (VOCs) in Liverpool Bay, Irish Sea. Marine Pollution Bulletin, 54(11), 1742-1753.

Cai, Q.-Y., Mo, C.-H., Wu, Q.-T., Zeng, Q.-Y., \& Katsoyiannis A. (2007) Occurrence of organic contaminants in sewage sludges from eleven wastewater treatment plants, China. Chemosphere, 68, 1751-1762.

de Zeeuw, R. A., Franke, J. P., Maurer, H. H., \& Pfleger, K. (1992). Gas chromatographic retention indices of toxicologically relevant substances on packed or capillary columns with dimethylsillicone stationary phases (2nd ed.). Weinheim: VCH Verlag.

Domeño, C., Martínez-García, F., Campo, L., \& Nerín, C. (2004). Sampling and analysis of volatile organic pollutants emitted by an industrial stack. Analytica Chimica Acta, 524, 51-62.

Eitzer, B. D. (1995). Emissions of volatile organic chemicals from municipal solid waste composting facilities. Environmental Science and Technology, 29, 896-902.

Escalasa, L., Guadayola, J. M., Cortinab, M., Riverab, L., \& Caixach, J. (2003). Time and space patterns of volatile organic compounds in a sewage treatment plant. Water Research, 37, 3913-3920.

Fang, J., Lovanh, N., \& Alvarez, P. J. (2004). The use of isotopic and lipid analysis techniques linking toluene degradation to specific microorganisms: Applications and limitations. Water Research, 38, 2529-2536.

Gu, Y.-Q., Mo, M.-H., Zhou, J. P., Zou, C. S., \& Zhang, K. Q. (2007). Evaluation and identification of potential organic nematicidal volatiles from soil bacteria. Soil Biology and Biochemistry, 39(10), 2567-2575.

Harrison, E. Z., Oakes, S. R., Hysell, M., \& Hay, A. (2006). Organic chemicals in sewage sludges. Science of the Total Environment, 367, 481-497. 
Hippelein, M. (2006). Analysing selected VVOCs in indoor air with solid phase microextraction (SPME): A case study. Chemosphere, 65, 271-277.

Isidorov, V. A., Kotowska, U., \& Vinogorova, V. T. (2005a). GC identification of organic compounds based on partition coefficients of their TMS derivatives in a hexane-acetonitrile system and retention indices. Analytical Sciences, 21, 483-489.

Isidorov, V., Vinogorova, V., \& Rafałowski, K. (2005b). Gas chromatographic determination of extractable compounds composition and emission rate of volatile terpenes from larch needle litter. Journal of Atmospheric Chemistry, 50(3), 263-273.

ISO (2000). ISO EN 16017-1: Air quality-sampling and analysis of volatile organic compounds in ambient air, indoor air and workplace air by sorbent tubel thermal desorption/capillary gas chromatography. Part 1: Pumped sampling.

ISO (2003). ISO EN 16017-2: Air quality-sampling and analysis of volatile organic compounds in ambient air, indoor air and workplace air by sorbent tubel thermal desorption/capillary gas chromatography. Part 2: Diffusive sampling.

Janssen, A., Ma, S., Lens, P., \& Lettinga, G. (1997). Performance of a sulfide-oxidizing expanded-bed reactor supplied with dissolved oxygen. Biotechnology and Bioengineering, 53, 32-40.

Jönsson, S., Gustavsson, L., \& van Bavel, B. (2007). Analysis of nitroaromatic compounds in complex samples using solid-phase microextraction and isotope dilution quantification gas chromatography-electron-capture negative ionisation mass spectrometry. Journal of Chromatography A, 1164, 65-73.

Kim, K.-H., Choi, Y. J., Jeon, E. C., \& Sunwoo, Y. (2005). Characterization of malodorous sulfur compounds in landfill gas. Atmospheric Environment, 39, 1103-1112.

Koe, L. C. C., \& Shen, W. (1997). High resolution GC-MS analysis of VOCs in wastewater and sludge. Environmental Monitoring and Assessment, 44, 549-561.

Llompart, M., García-Jares, C., Salgado, C., Polo, M., \& Cela R. (2003). Determination of musk compounds in sewage treatment plant sludge samples by solid-phase microextraction. Journal of Chromatography A, 999, 185-193.

Llop, A., Borrull, F., \& Pocurull, E. (2010). Pressurised hot water extraction followed by simultaneous derivatization and headspace solid-phase microextraction and gas chromatography-tandem mass spectrometry for the determination of aliphatic primary amines in sewage sludge. Analytica Chimica Acta, 665, 231-236.

Lu, R., Wu, J., Turco, R. P., Winer, A. M., Atkinson, R., Arey, J., et al. (2005). Naphthalene distributions and human exposure in Southern California. Atmospheric Environment, 39, 489-507.

Mangani, G., Berloni, A., \& Maione, M. (2003). "Cold" solid-phase microextraction method for the determination of volatile halocarbons present in the atmosphere at ultra-trace levels. Journal of Chromatography A, 988, 167-175.

Matysik, S., Herbarth, O., \& Mueller, A. (2009) Determination of microbial volatile organic compounds
(MVOCs) by passive sampling onto charcoal sorbents. Chemosphere, 76, 114-119.

NSAI (2011). prEN 13649: Stationary source emissionsdetermination of the mass concentration of individual gaseous organic compounds - test methods. Dublin: NSAI.

Pagans, E., Front, X., \& Sanchez A. (2006). Emission of volatile organic compounds from composting of different solid wastes: Abatement by biofiltration. Journal of Hazardous Materials, 131, 179-186.

Parke, D. V., \& Lewis, D. F. (1992). Safety aspects of food preservatives. Food Additives and Contaminants, 9(5), 561-577.

Pawliszyn, J. (1997). Solid-phase microextraction, theory and practice. New York: Wiley.

Pawliszyn, J., \& Liu, S. (1987). Sample introduction for capillary gas chromatography with laser desorption and optical fibers. Analytical Chemistry, 59, 1475-1478.

Sadtler Research Laboratory (1986). Standard GC retention index library. Philadelphia: Sadtler Research Laboratory.

Safer, A. M., \& al-Nughamish, A. J. (1999). Hepatotoxicity induced by the anti-oxidant food additive, butylated hydroxytoluene (BHT), in rats: An electron microscopical study. Histology and Histopathology, 14, 391406.

Simonich, S. L., Begley, W. M., Debaere, G., \& Eckhoff, W. S. (2000). Trace analysis of fragrance materials in wastewater and treated wastewater. Environmental Science and Technology, 34, 959-965.

Spinhirne, J. P., Koziel, J. A., \& Chirase, N. K. (2004). Sampling and analysis of volatile organic compounds in bovine breath by solid-phase microextraction and gas chromatography-mass spectrometry. Journal of Chromatography A, 1025, 63-69.

Tryphonas, H., Lacroix, F., Lok, E., Jee, P. D., Clayson, B., Hayward, S., et al. (1999). The effect of butylated hydroxytoluene on selected immune surveillance parameters in rats bearing enzyme-altered hepatic preneoplastic lesions. Food and Chemical Toxicology, 37(7), 671-681.

US EPA (1996). US EPA Method 5041a: Desorption and analysis of sorbent cartridges from volatile organic sampling train (VOST). Washington, DC: US EPA.

Van den Dool, H., \& Kratz, P. (1963). A generalization of retention index system including linear temperature programmed gas-liquid partition chromatography. Journal of Chromatography, 11, 463471.

Van Durme, G. P., McNamara, B. F., \& McGinley, C. M. (1992). Bench-scale removal of odor and volatile organic compounds at a composting facility. Water Environment Research, 64, 19-27.

Villaverde, M. L., Juárez, M. P., \& Mijailovsky, S. (2007). Detection of Tribolium castaneum (Herbst) volatile defensive secretions by solid phase microextractioncapillary gas chromatography (SPME-CGC). Journal of Stored Products Research, 43(4), 540-545.

Webber, M. D., Rogers, H. R., Watts, C. D., Boxall, A. B. A., Davis, R. D., \& Scoffin, R. (1996). Monitoring and prioritisation of organic contaminants 
in sewage sludges using specific chemical analysis and predictive, non-analytical methods. Science of the Total Environment, 185, 27-44.

Wilson, S. C., Duarte-Davidson, R., \& Jones, K. C. (1996a). Screening the environmental fate of organic contaminants in sewage sludges applied to agricultural soils: 1. The potential for downward movement to groundwaters. Science of the Total Environment, 185, 45-57.

Wilson, S. C., Duckham, C., \& Jones, K. C. (1996b). Quantification of volatile organic compound losses from sludge amended soil: A pilot scale study. Chemosphere, 33, 1261-1272.

Woolfenden, E. (2010a). Sorbent-based sampling methods for volatile and semi-volatile organic compounds in air. Part 1: Sorbent-based air monitoring options. Journal of Chromatography A, 1217, 2674-2684.

Woolfenden, E. (2010b). Sorbent-based sampling methods for volatile and semi-volatile organic compounds in air. Part 2: Sorbent-based air monitoring options. Journal of Chromatography A, 1217, 2685-2694.

Wu, S.-F., \& Ding, W.-H. (2010) Fast determination of synthetic polycyclic musks in sewage sludge and sediments by microwave-assisted headspace solid-phase microextraction and gas chromatography-mass spectrometry. Journal of Chromatography A, 1217, 27762781.

Wu, B.-Z., Feng, T.-Z., Sree, U., Chiu, K.-H., \& Lo, J.G. (2006). Sampling and analysis of volatile organics emitted from wastewater treatment plant and drain system of an industrial science park. Analytica Chimica Acta, 576, 100-111.

Zeng, J., Chen, J., Chen, L., Wang, Y., Chen, W., Huang, X., et al. (2009). The extraction performance of methacrylic acid-trimethylolpropanetrimethacrylate solid-phase microextraction fibers in aqueous solutions. Analytica Chimica Acta, 648, 194-199. 